\title{
Effects of Hand Washing Campaign on Dynamical Model of Hand Foot Mouth Disease
}

\author{
Thanyada Phutthichayanon and Surapol Naowarat
}

\begin{abstract}
In this study, we proposed a nonlinear mathematical model of Hand Foot Mouth Disease (HFMD) due to the effectiveness of hand washing campaign as a control strategy. The model is analyzed using stability theory of differential equations and computer simulation. The results showed that there were two equilibrium points; disease free equilibrium and endemic equilibrium point. The qualitative results depend on the basic reproductive number $\left(R_{0}\right)$. We obtained the basic reproductive number by using the next generation method. Stabilities of the model are determined by Routh - Hurwitz criteria. If $R_{0}<1$, then the disease free equilibrium point is local asymptotically stable, but If $R_{0}>1$, then the endemic equilibrium point is local asymptotically stable. The graphical representations are provided to qualitatively support the analytical results. It concluded that with an increase in the effectiveness of hand washing campaign, the infected population reduced.
\end{abstract}

Index Terms-Hand foot mouth disease, hand washing campaign, basic reproductive number, stability analysis, equilibrium point.

\section{INTRODUCTION}

Hand Foot Mouth Disease (HFMD) is a common infectious disease that affects infants and children. HFMD is caused by an Enterovirus genus of Piconoviridae family [1]. The most common viruses causing HFMD are Coxsackievirus A16 (COX A16) and Enterovirus71 (EV71)[2]. Symptoms of HFMD is usually onset a fever, poor appetite, malaise, and sore throat. After fever starts, painful sores can develop in the mouth. The skin rush with flat or raised red spots can develop on the palms of the hands and soles of the feet and sometimes on the buttocks. Although, HFMD is a moderate contagious and not a serious illness among population [3]. There is no specific treatment or vaccine for HFMD. Therefore, the control measures of HFMD are based on appropriate prevention measure include quarantine mechanisms and personal protection against exposure to infected persons [4]. In Asia, HFMD is occurred in many countries; Malasia (1997 and 2006), Taiwan(1998), China(2008, 2009 and 2010), Singapore(2008), Vietnam (2008) and Mongolia(2008), Bruni(2008),Indonesia(2009) and Thailand(1958 and 2008) [3].

Mathematical models have become an important tool for understanding the spread and control of disease. Reference [3]. proposed a simple SEIR model for HFMD among the

\footnotetext{
Manuscript received December 9, 2014; revised March 4, 2015. This work was supported in part by Faculty of Science and Technology, Suratthani Rajabhat University,Surat Thani, 84100, Thailand.

The authors are with Faculty of Science and Technology, Suratthani Rajabhat University, Surat Thani, 84100, Thailand (e-mail: na_math_sru@hotmail.com, su1963n@gmail.com).
}

young children. The aim of this study to understand the dynamics of HFMD. The model was analyzed for analytic results and numerical simulation results. The results shown that disease transmission depends on the number of actively infected human at the initial time and also on the disease transmission coefficient at the given time. Reference [4] proposed an epidemic model of HFMD with periodic transmission rate. HFMD model was analyzed and investigated the effects of quarantine in children population. We obtain a threshold value which determines the extinction and uniform persistence of the disease. The results show that disease free equilibrium is global asymptotically stable if the threshold value is less than one. Otherwise, the system has a positive periodic solution and disease persist. The simulation results show that quarantine is beneficial intervention to control for this disease. Reference [5] proposed a mathematical model of HFMD to understand the dynamics and analyze the effectiveness of quarantine as a control strategy. The results show that disease could be control by quarantine of more actively infected individuals. The qualitative results show that disease transmission depends more on the number of actively infected at the initial time and also on the disease transmission coefficient at a given time. Reference [6] proposed a dynamic model with periodic transmission rates to investigate the seasonal HFMD. We obtain the basic reproductive number, analyze the dynamical behavior of the model and simulate the HFMD of Shandong Province. By carrying out the sensitivity analysis of some key parameters, we conclude that the recessive subpopulation plays an important role in the spread of HFMD.

The objective of the study is to determine the effectiveness of hand washing campaign as a control strategy on the dynamical transmission of HFMD model. The remainder of the paper is organized as follows. In Section II, we formulate the propose model. In Section III, we analyze the model by using stability theory of differential equations, to determine both disease free and endemic equilibrium point, derive the basic reproductive number and investigate the stability of the model. In Section IV, we simulate the numerical results, which confirm our theoretical results. Finally, we discussion and conclude our study in Section V.

\section{MODEL FORMULATION}

In our model, we classified the population into five compartments according to their states: the susceptible human (S), the exposed human (E), the infected human (I), the severe infected human $\left(I_{A}\right)$ and the recovered human (R), We denote the total population by $\left(N_{T}\right)$.The dynamics transmission of disease associated with these compartments are illustrated as shown in Fig. 1. 


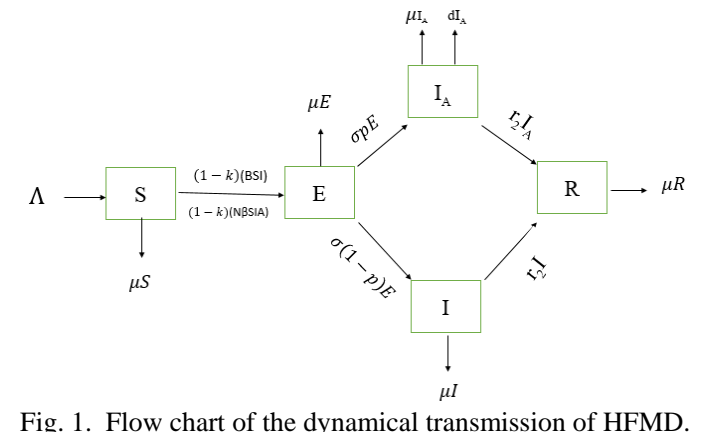

Fig. 1. Flow chart of the dynamical transmission of HFMD.

We defined,

$S$ is the number of susceptible human population at time $t$

$E$ is the number of exposed human population at time $t$

$I$ is the number of infected human population at time $t$

$I_{A}$ is the number of severe infected human population at time $t$

$R$ is the number of recovered human population at time $t$

The transmission model will be a system of ordinary differential equations given by,

$$
\begin{gathered}
\frac{d S}{d t}=\Lambda-(1-k)\left(B S I+n \beta S I_{A}\right)-\mu S \\
\frac{d E}{d t}=(1-k)\left(B S I+n \beta S I_{A}\right)-\sigma(1-p) E-\sigma p E-\mu E \\
\frac{d I}{d t}=\sigma(1-p) E-r_{2} I_{A}-\mu I \\
\frac{d I_{A}}{d t}=\sigma p E-r_{1} I_{A}-\mu I_{A}-d I_{A} \\
\frac{d R}{d t}=r_{2} I_{A}-r_{2} I-\mu R
\end{gathered}
$$

where

$\Lambda$ is the birth rate of human population,

$\mu$ is the natural death rate of human population,

$\beta$ is the probability of transmission,

$n$ is the number of contacts,

$\frac{1}{\sigma}$ is the average of incubation period,

$r_{1}$ is the recovery rate of severe infected human,

$r_{2}$ is the recovery rate of infected human,

$p$ is the fraction of developing infected cases,

$d$ is the infected disease-related death rate,

$k$ is the effectiveness of hand washing campaign, with

$$
N_{T}=S+E+I+I_{A}+R
$$

By adding (1)-(5), we obtain

$$
\frac{d N_{T}}{d t}=\wedge-\mu N_{T}-\mu I_{A}
$$

\section{MODEL ANALYSIS}

\section{A. Equilibrium Points}

Disease free equilibrium point $\left(E_{0}\right)$ : there are no infected human and severe infected human, that is $I=0, I_{A}=0$. Substituting $\quad I=0, I_{A}=0 \quad$ in (1)-(7), we obtained $E_{0}\left(S, E, I, I_{A}, N_{T}\right)=E_{0}\left(\frac{\Lambda}{\mu}, 0,0,0, \frac{\Lambda}{\mu}\right)$.

Endemic equilibrium point $\left(E_{1}\right)$ : In case $I^{*}>0, I_{A}^{*}>0$.

We obtained,

$E_{1}\left(S^{*}, E^{*}, I^{*}, I_{A}^{*}, N_{T}^{*}\right)=$

$\left(\frac{\Lambda}{(1-k)\left(\beta I^{*}+n \beta I_{A}^{*}\right)+\mu}, \frac{(1-k)\left(\beta I^{*}+n \beta I_{A}^{*}\right) \Lambda}{(\sigma+\mu)(1-k)\left(\beta I^{*}+n \beta I_{A}^{*}\right)+\mu}, I^{*}, I_{A}^{*}, \frac{\Lambda-d I_{A}^{*}}{\mu}\right)$ where the value of $I^{*}$ is a positive root of cubic equation,

$$
H_{1} I^{* 3}+H_{2} I^{* 2}+H_{3} I^{*}+H_{4}=0
$$

with

$$
\begin{gathered}
H_{1}=A_{2}^{2} A_{6}-A_{2} A_{7} A_{3}, \\
H_{2}=\left(A_{1} A_{7}-A_{3} A_{8}\right) A_{3}+A_{2} A_{4} A_{7}-\left(A_{1} A_{6}-A_{3} A_{5}+A_{1} A_{6}\right) A_{2}, \\
H_{3}=A_{4} A_{8} A_{3}-\left(A_{1} A_{7}-A_{3} A_{8}\right) A_{4}+\left(A_{3} A_{5}-A_{1} A_{6}\right) A_{1}+A_{4} A_{5} A_{2}, \\
H_{4}=A_{1} A_{4} A_{5}+A_{4}^{2} A_{8}, \\
A_{1}=\sigma \Lambda \beta(1-p)(1-k)-\left(r_{2}+\mu\right) \mu, A_{2}=\left(r_{2}+\mu\right)(\sigma+\mu)(1-k) \beta, \\
A_{3}=\left(r_{2}+\mu\right)(\sigma+\mu)(1-k) n \beta, A_{4}=\sigma n \Lambda \beta(1-p)(1-k), \\
A_{5}=\sigma p(1-k) n \Lambda \beta-\mu\left(r_{1}+\mu+d\right), A_{6}=\left(r_{1}+\mu+d\right)(\sigma+\mu)(1-k) n \beta, \\
A_{7}=\left(r_{1}+\mu+d\right)(\sigma+\mu)(1-k) \beta, A_{8}=\sigma p(1-k) \Lambda \beta .
\end{gathered}
$$

And the value of $I_{A}^{*}$ is a positive root of cubic equation,

$$
M_{1} I_{A}^{* 3}+M_{2} I_{A}^{* 2}+M_{3} I_{A}^{*}+M_{4}=0
$$

where

$M_{1}=\left(A_{2} A_{6}-A_{3} A_{7}\right) A_{6}$

$M_{2}=\left(A_{3} A_{5}-A_{4} A_{7}\right) A_{7}+\left(A_{1} A_{7}-2 A_{2} A_{5}+A_{3} A_{8}\right) \mathrm{A}_{6}$,

$M_{3}=A_{4} A_{7} A_{8}-A_{1} A_{6} A_{8}-\left(A_{1} A_{7}-A_{2} A_{5}\right) A_{5}-\left(A_{3} A_{5}-A_{4} A_{7}\right) A_{8}$,

$M_{4}=A_{1} A_{5} A_{8}-A_{4} A_{8}^{2}$.

Basic Reproductive number: We obtained a basic reproductive number $\left(R_{0}\right)$ by using the next generation matrix [7]. Rearrange the (1) - (4) and (7) in matrix form

$$
\begin{gathered}
F(x)=\left[\begin{array}{c}
0 \\
(1-k)\left(\beta S I+n \beta S I_{A}\right) \\
0 \\
0 \\
0
\end{array}\right], V(x)=\left[\begin{array}{c}
(1-k)\left(\beta S I+n \beta S I_{A}\right)+\mu S-\Lambda \\
\sigma(1-p) E+\sigma p E+\mu E \\
r_{2} I+\mu I-\sigma(1-p) E \\
r_{1} I_{A}+\mu I_{A}+d I_{A}-\sigma p E \\
\mu N_{T}+d I_{A}-\Lambda
\end{array}\right] \\
\frac{d x}{d t}=F(x)-V(x)
\end{gathered}
$$

where $F(x)$ is the rate of appearance of new infections in compartment and $V(x)$ is the transfer of individuals out of compartment by all other means. Find the Jacobian of $F(x)$ and $V(x)$, denoted by $D F(x)=F$ and $D V(x)=V$, we obtained

$$
F=\left[\begin{array}{ccccc}
0 & 0 & 0 & 0 & 0 \\
(1-k)\left(\beta I+n \beta I_{A}\right) & 0 & (1-k) \beta S & (1-k) n \beta S & 0 \\
0 & \mathrm{p} & 0 & 0 & 0 \\
0 & 0 & 0 & 0 & 0 \\
0 & 0 & 0 & 0 & 0
\end{array}\right]
$$


and

$$
V=\left[\begin{array}{ccccc}
(1-k)\left(\beta I+n \beta I_{A}\right)+\mu & 0 & (1-k) \beta S & (1-k) n \beta S & 0 \\
0 & \sigma+\mu & 0 & 0 & 0 \\
0 & -\sigma(1-p) & r_{2}+\mu & 0 & 0 \\
0 & -\sigma p & 0 & r_{1}+\mu+d & 0 \\
0 & 0 & d & 0 & \mu
\end{array}\right]
$$

Find $F$ and $V$ at $E_{0}\left(S, E, I, I_{A}, N_{T}\right)=E_{0}\left(\frac{\Lambda}{\mu}, 0,0,0, \frac{\Lambda}{\mu}\right)$

We obtained,

$$
\begin{aligned}
& F=\left[\begin{array}{ccccc}
0 & 0 & 0 & 0 & 0 \\
0 & 0 & (1-k) \beta \frac{\Lambda}{\mu} & (1-k) n \beta \frac{\Lambda}{\mu} & 0 \\
0 & p & 0 & 0 & 0 \\
0 & 0 & 0 & 0 & 0 \\
0 & 0 & 0 & 0 & 0
\end{array}\right] \text { and } \\
& V=\left[\begin{array}{ccccc}
\mu & 0 & (1-k) \beta \frac{\Lambda}{\mu} & (1-k) n \beta \frac{\Lambda}{\mu} & 0 \\
0 & \sigma+\mu & 0 & 0 & 0 \\
0 & -\sigma(1-p) & \mathrm{r}_{2}+\mu & 0 & 0 \\
0 & -\sigma p & 0 & r_{1}+\mu+d & 0 \\
0 & 0 & d & 0 & \mu
\end{array}\right]
\end{aligned}
$$

Find $F V^{-1}$, we get

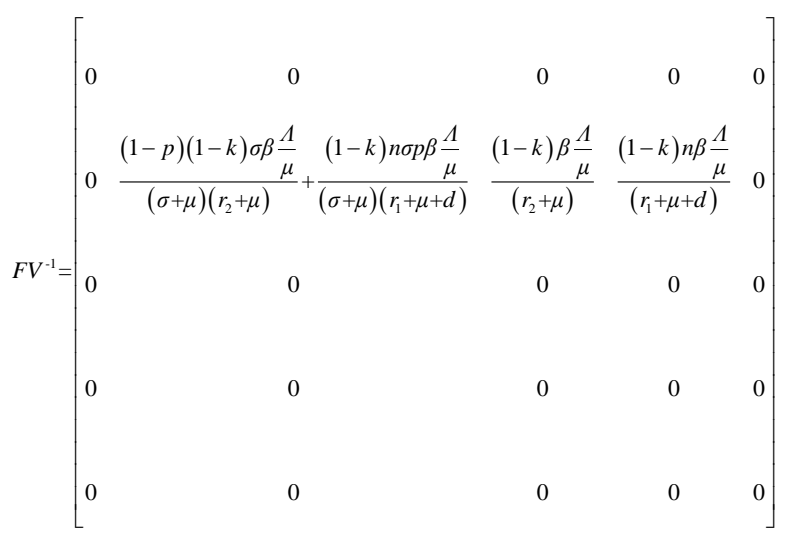

Find the spectral radius of $F V^{-1}$ denoted by $\rho\left(F V^{-1}\right)$

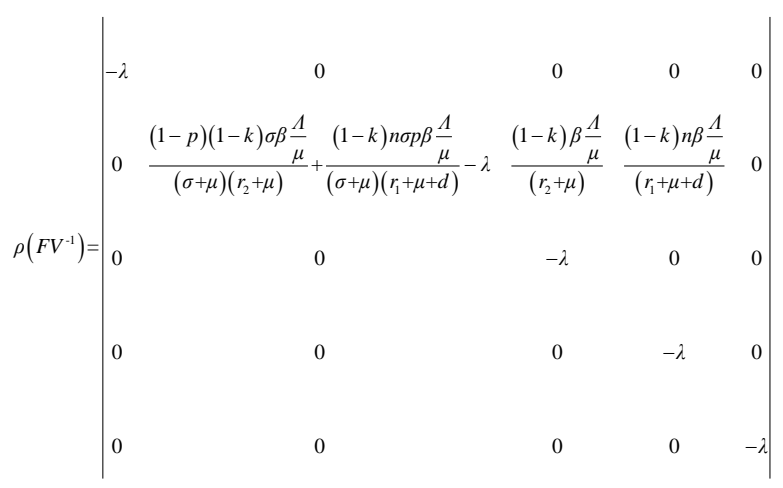

We obtain, the basic reproductive number as follow:

$$
R_{0}=\sqrt{\frac{(1-p)(1-k) \sigma \beta \frac{\Lambda}{\mu}}{(\sigma+\mu)\left(r_{2}+\mu\right)}+\frac{(1-k) n \sigma p \beta \frac{\Lambda}{\mu}}{(\sigma+\mu)\left(r_{1}+\mu+d\right)}}
$$

Local Stability: The local stability of an equilibrium point is determined from the Jacobian matrix of the system of ordinary differential equation (1)-(4) and (6) evaluated at the equilibrium point. The Jacobian matrix at $E_{0}$ is

$$
J_{0}=\left[\begin{array}{ccccc}
-\mu & 0 & -(1-k) \beta S & -(1-k) n \beta \frac{\grave{U}}{\mu} & 0 \\
0 & -\sigma-\mu & (1-k) \beta \frac{\grave{U}}{\mu} & (1-k) n \beta \frac{\grave{U}}{\mu} & 0 \\
0 & \sigma(1-p) & -r_{2}-\mu & 0 & 0 \\
0 & \sigma p & 0 & -r_{1}-\mu-d & 0 \\
0 & 0 & 0 & -d & -\mu
\end{array}\right]
$$

The eigenvalues of the $J_{0}$ are obtained by solving equation $\left|\mathrm{J}_{0}-\lambda \mathrm{I}\right|=0$. We obtained the characteristic equation,

$$
(\lambda+\mu)^{2}\left(\lambda^{3}+X_{1} \lambda^{2}+X_{2} \lambda+X_{3}\right)=0
$$

where

$$
\begin{aligned}
& X_{1}=C_{0}+r_{1}+r_{2}+2 \mu, C_{0}=\sigma+\mu \\
& X_{2}=\left(r_{1}+\mu\right)\left(C_{0}+r_{2}+\mu\right)+\left(r_{2}+\mu\right) C_{0}-\sigma \beta \frac{\grave{U}}{\mu}(1-k)(n p+1-p), \\
& X_{3}=\left(r_{1}+\mu\right)\left(r_{2}+\mu\right) C_{0}-\left(\left(r_{2}+\mu\right) n p+\left(r_{1}+\mu+d\right)(1-p)\right)(1-k) \sigma \beta \frac{\grave{U}}{\mu} .
\end{aligned}
$$

From the characteristic equation, We see that two are $\lambda_{1}=-\mu<0, \lambda_{2}=-\mu<0$. The other three eigenvalues are solution of $\lambda^{3}+X_{1} \lambda^{2}+X_{2} \lambda+X_{3}=0$. The roots of this equation will be negative if two coefficients satisfied with the Routh-Hurwitz criteria [8].

$$
\text { 1) } X_{1}>0, \text { 2) } X_{3}>0, \text { 3) } X_{1} X_{2}>X_{3} \text {. }
$$

Endemic equilibrium point: To determine the stability of the endemic equilibrium point, $E_{1}$ by examining the eigenvalues of Jacobian matrix at $E_{1}$, which is

$J_{1}=\left[\begin{array}{ccccc}-(1-k)\left(\beta I^{*}+n \beta I_{A}{ }^{*}\right)-\mu & 0 & -(1-k) \beta S^{*} & -(1-k) n \beta S^{*} & 0 \\ (1-k)\left(\beta I^{*}+n \beta I_{A}{ }^{*}\right) & -(\sigma+\mu) & (1-k) \beta S^{*} & (1-k) n \beta S^{*} & 0 \\ 0 & \sigma(1-p) & -r_{2}-\mu & 0 & 0 \\ 0 & \sigma p & 0 & -r_{1}-\mu-d & 0 \\ 0 & 0 & 0 & -\mathrm{d} & -\mu\end{array}\right]$ and by solving equation $\left|J_{1}-\lambda I\right|=0$. We obtain the characteristic equation,

$$
(\lambda+\mu)\left(\lambda^{4}+Q_{1} \lambda^{3}+Q_{2} \lambda^{2}+Q_{3} \lambda+Q_{4}\right)=0
$$

where

$$
\begin{aligned}
& Q_{1}=A+C+G+J, Q_{2}=K+(C+G+J) A \\
& Q_{3}=A K+(H+F) B D-L, Q_{4}=(F J+H G) B D-A L
\end{aligned}
$$




$$
\begin{gathered}
A=(1-k)\left(\beta I^{*}+n \beta I_{A}^{*}\right)+\mu, B=(1-k)\left(\beta I^{*}+n \beta I_{A}^{*}\right), C=\sigma+\mu, \\
D=\sigma p, F=(1-k) \beta S^{*}, G=r_{2}+\mu, J=r_{1}+\mu+d, H=(1-k) n \beta S^{*} \\
K=C G+(C+G) J-(H+F) D, L=-C G J+(F J+G H) D .
\end{gathered}
$$

We obtain the eigenvalues are $\lambda_{1}=-\mu<0$, and the remaining four eigenvalues of $\lambda^{4}+Q_{1} \lambda^{3}+Q_{2} \lambda^{2}+Q_{3} \lambda+Q_{4}=0$ will be negative real part if they satisfy the Routh - Hurwitz criteria [9] as follows.

$$
\begin{aligned}
& \text { 1) } \left.Q_{1}>0 \text {, 2) } Q_{1} Q_{2}-Q_{3}>0,3\right) Q_{1}\left(Q_{2} Q_{3}+Q_{1} Q_{4}\right)-Q_{3}^{2}>0 \text {, } \\
& \text { 4) } Q_{4}\left(-Q_{3}^{2}+Q_{1} Q_{2} Q_{3}-Q_{1}^{2} Q_{4}\right)>0
\end{aligned}
$$

\section{NUMERICAL RESULTS}

In this study, we are interested in the transmission model of HFMD with the effectiveness of hand washing campaign. The system is simulated for various set of parameters. The stability of disease free equilibrium point $\left(E_{0}\right)$ and endemic equilibrium point $\left(E_{1}\right)$ are shown in Fig. 2 and Fig. 3, respectively. The values of parameters used for simulation in this model as shown in Table I.

TABLE I: PARAMETER VALUES USED IN NUMERICAL SIMULATION

\begin{tabular}{|c|l|l|}
\hline Parameters & \multicolumn{1}{|c|}{ Description } & \multicolumn{1}{c|}{ Values } \\
\hline$\mu$ & $\begin{array}{l}\text { Birth rate of human } \\
\text { population }\end{array}$ & $\begin{array}{l}100000 /(420) \text { per } \\
\text { week }\end{array}$ \\
\hline $\begin{array}{c}\text { Natural death rate of human } \\
\text { population }\end{array}$ & $1 /(420)$ per week \\
\hline$\beta$ & Probability of transmission & 0.00007 \\
\hline$\sigma$ & Number of contacts & 0.00001 per week \\
\hline$r_{1}$ & $\begin{array}{l}\text { Recovery rate of severe } \\
\text { infected human }\end{array}$ & 0.8235 per week \\
\hline$r_{2}$ & $\begin{array}{l}\text { Recovery rate of infected } \\
\text { human per week }\end{array}$ & 0.8235 per week \\
\hline$p$ & $\begin{array}{l}\text { Fraction of developing } \\
\text { infected cases }\end{array}$ & 0.025 per week \\
\hline$d$ & $\begin{array}{l}\text { Death rate related infected } \\
\text { disease }\end{array}$ & 0.01 per week \\
\hline$k$ & $\begin{array}{l}\text { Effectiveness of hand washing } \\
\text { campaign }\end{array}$ & 0.90 \\
\hline
\end{tabular}

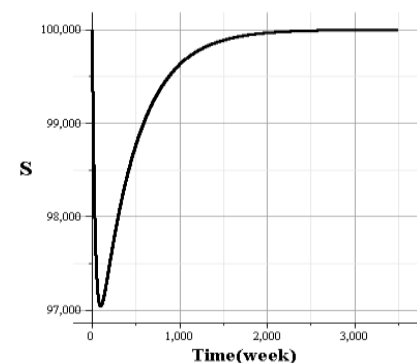

(a)

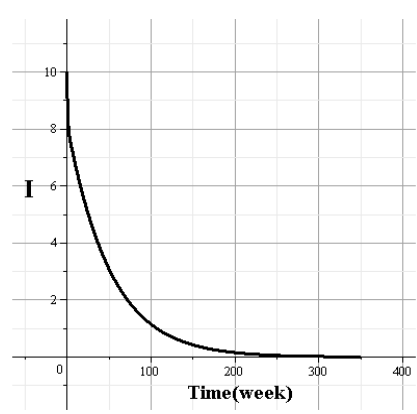

(b)

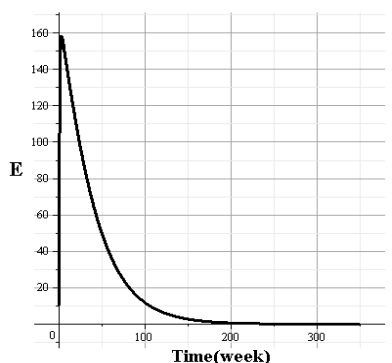

(b)

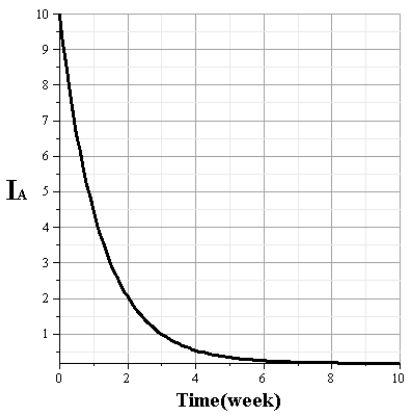

(d)

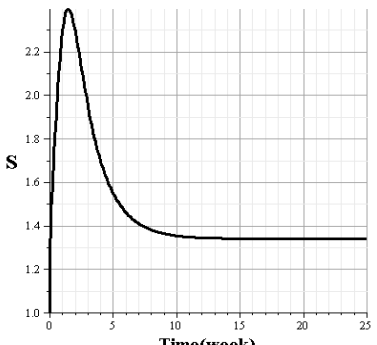

(a)

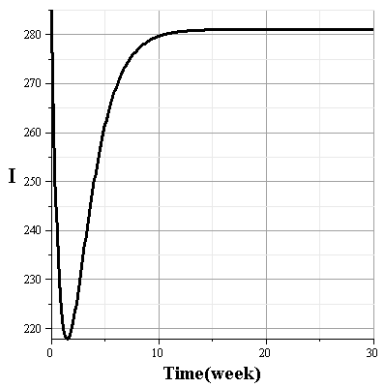

(c)

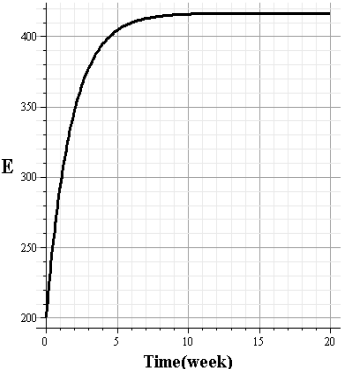

(b)

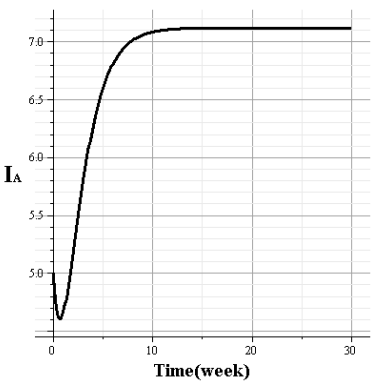

(d) 


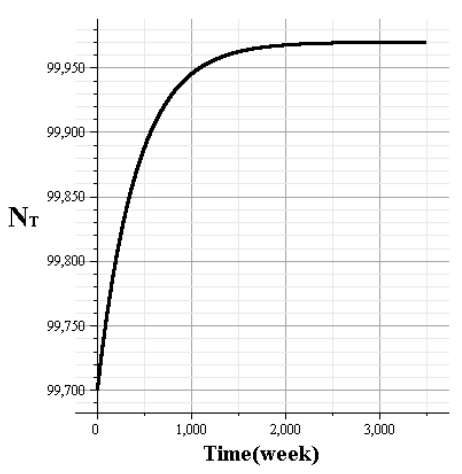

(e)

Fig. 3. Time series of (a) Susceptible human (S), (b) Exposed human (E), (c) Infected human (I), (d) Severe Infected human $\left(I_{A}\right)$, and (e) Total human $\left(N_{T}\right)$ proportion approach to the endemic equilibrium state $E_{1}$.

\section{CONCLUSION}

We formulate the transmission model of HFMD by incorporating the effect of hand washing campaign to protect individual from HFMD. The basic reproductive number is $\Re_{0}=\sqrt{R_{0}}$, where

$$
R_{0}=\frac{(1-p)(1-k) \sigma \beta \frac{\Lambda}{\mu}}{(\sigma+\mu)\left(r_{2}+\mu\right)}+\frac{(1-k) n \sigma p \beta \frac{\Lambda}{\mu}}{(\sigma+\mu)\left(r_{1}+\mu+d\right)}
$$

Hence, $R_{0}$ represent the average number of secondary infections produce when one infected individual is introduced into a host population where everyone is susceptible [10]. In addition, $\mathrm{R}_{0}$ is the threshold condition for determining the stability of the system. If $R_{0}<1$, the disease free equilibrium point is local asymptotically stable as shown in Fig. 2, that is disease will die out from the community. If $R_{0}>1$, the endemic equilibrium point is local asymptotically stable as shown in Fig. 3, that is the disease will persist in the community. For the endemic state the value is greater than one due to young children do not know how to protect themselves from the infected children [1].

We can conclusion that if each individual who live in the community has knowledge, attitude and behavior to protect them from HFMD by increasing the effectiveness of hand washing campaign. The number of susceptible human to contact the HFMD will decrease. In young children school or nursery and kindergarten, as HFMD has occurred. This alternative intervention could decrease the number of infected human by hand washing campaign for all young students at schooldays.

\section{ACKNOWLEDGEMENT}

Surapol Naowarat would like to thank Department of Mathematics, Faculty of Science and Technology, Suratthani Rajabhat University, Surat Thani, Thailand for financial support.

\section{REFERENCES}

[1] CDC. (2014) About hand, foot, and mouth disease. [Online]. Available: http://www.cdc.gov/hand-foot-mouth.

[2] Z. Qi, H. Y. Tao, M. J. Qi,Y. S. Cheng, and W. Yu, "Surveillance of hand, foot, and mouth disease in mainland china (2008-2009)," Biomed Environ Sci., vol. 24, no. 4, pp. 349-356, June 2011.

[3] N. Roy and N. Halder, "Compartmental Modeling of Hand, Foot and Mouth Infectious Disease (HFMD)," Research Journal of Applied Sciences, vol. 5, no. 3, pp. 177-182, 2010.

[4] J. Lui, "Threshhold dynamics for a HFMD epidemic model with periodic transmission rate," Nonlinear Dyn, vol. 64, pp. 89-95, 2011.

[5] N. Roy, "Mathematical modelling of hand-foot-Mouth Disease: quarantine as a control measure," Internationals Journal of Advanced Scientific Engineering and Technological Research, vol. 1, no. 2, pp. 34-44, 2012.

[6] Y. MA, M. Liu, Q. Hou, and J. Zhao, "Modelling seasonal HFMD with the recessive infection in shandong, China," Math Biosci Eng, vol. 10 , no. 3 , pp. 1159-1171, 2013 .

[7] P. Van D. Driessche and J Watmough, Reproductive numbers and sub-threshold endemic equilibria for compartment models of disease transmission," Math. Biosci, vol. 180, pp. 29-48, 2002.

[8] L. J. S. Allen, An Introduction to Mathematical Biology, Pearson/Prince Hall, Upper Saddle River, New Jersey, 2006.

[9] J. E. Marsden and M. McCracken, The Hoft Bifurcationand Its Applications, Springer-Verlag, New York, 1976.

[10] R. M. Anderson and R. M. May, Infectious Diseases in Human: Dynamics and Control, Oxford University Press, Oxford, 1991.

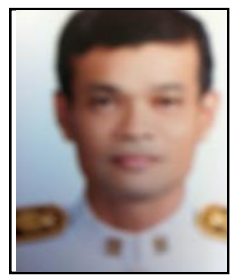

Surapol Naowarat was born in Phatthalung, Thailand in 1963. He has obtained the B.Sc. in mathematics from Prince of Songkhla University, Pattani, Thailand in 1984 after that he has obtained M. Ed. in mathematics education from Chulalongkon University, Bangkok, Thailand in 1993 and he received his Ph.D. in mathematics from Mahidol University, Bangkok, Thailand in 2004. He is an assistant professor at Department of Mathematics, Faculty of Science and Technology, Surathani Rajabhat University, Sura Thani, Thailand. He is interested in the research about mathematical model of disease transmission.

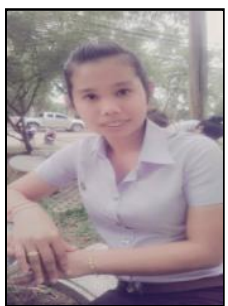

Thanyada Phutthichayanon was born in Surat Than province, Thailand in 1991. She has obtained the B.Sc. in mathematics from Suratthani Rajabhat University, Surat Thani, Thailand in 2014. She is a teacher in secondary school. 